\title{
Methylation studies in Peromyscus: aging, altitude adaptation, and monogamy
}

\author{
Steve Horvath (D) Amin Haghani · Joseph A. Zoller • Asieh Naderi • \\ Elham Soltanmohammadi • Elena Farmaki • Vimala Kaza • \\ Ioulia Chatzistamou $\cdot$ Hippokratis Kiaris
}

Received: 11 August 2021 / Accepted: 7 October 2021 / Published online: 26 October 2021

(C) The Author(s) 2021, corrected publication 2021

\begin{abstract}
DNA methylation-based biomarkers of aging have been developed for humans and many other mammals and could be used to assess how stress factors impact aging. Deer mice (Peromyscus) are long-living rodents that have emerged as an informative model to study aging, adaptation to extreme environments, and monogamous behavior. In the present study, we have undertaken an exhaustive, genomewide analysis of DNA methylation in Peromyscus, spanning different species, stocks, sexes, tissues, and age cohorts. We describe DNA methylation-based
\end{abstract}

Supplementary Information The online version contains supplementary material available at https://doi. org/10.1007/s11357-021-00472-5.

S. Horvath $(\bowtie) \cdot$ A. Haghani

Department of Human Genetics, David Geffen School of Medicine, University of California, Los Angeles, CA, USA

e-mail: shorvath@mednet.ucla.edu

A. Haghani

e-mail: ahaghani@g.ucla.edu

S. Horvath · J. A. Zoller

Department of Biostatistics, Fielding School of Public

Health, University of California, Los Angeles, CA, USA

e-mail: jaz18@g.ucla.edu

A. Naderi · E. Soltanmohammadi · E. Farmaki .

H. Kiaris $(\bowtie)$

Department of Drug Discovery and Biomedical Sciences,

College of Pharmacy, University of South Carolina,

Columbia, SC, USA

e-mail: hk@sc.edu estimators of age for different species of deer mice based on novel DNA methylation data generated on highly conserved mammalian CpGs measured with a custom array. The multi-tissue epigenetic clock for deer mice was trained on 3 tissues (tail, liver, and brain). Two human-Peromyscus clocks accurately measure age and relative age, respectively. We present $\mathrm{CpGs}$ and enriched pathways that relate to different conditions such as chronological age, high altitude, and monogamous behavior. Overall, this study provides a first step towards studying the epigenetic correlates of monogamous behavior and adaptation to high altitude in Peromyscus. The human-Peromyscus epigenetic clocks are expected to provide a significant

\author{
E. Soltanmohammadi \\ e-mail: elhams@email.sc.edu \\ E. Farmaki \\ e-mail: efarmaki@coh.org \\ V. Kaza $\cdot$ H. Kiaris \\ Peromyscus Genetic Stock Center, University of South \\ Carolina, Columbia, SC, USA \\ e-mail:kaza@email.sc.edu \\ I. Chatzistamou \\ Department of Pathology, Microbiology and Immunology, \\ School of Medicine, University of South Carolina, \\ Columbia, SC, USA \\ e-mail: chatzistamou@uscmed.sc.edu
}


boost to the attractiveness of Peromyscus as a biological model.

Keywords Deer mouse - Aging - Epigenetic clock · DNA methylation

\section{Introduction}

Cytosine methylation is an epigenetic mechanism which plays a critical role in mammalian development [1]. Methyl groups may help to generate a local chromatin configuration that renders genes inaccessible, and thus transcriptionally inactive [2]. In general, cytosine methylation at specific sites is influenced by both heritable as well as environmental factors [3]. It has long been recognized that age has a strong effect on DNA methylation (DNAm) levels [4-6]. DNA methylation data can be used to develop multivariate regression models that accurately estimate chronological age for any tissue across the entire lifespan of mammals [7-13]. These DNAm-based age estimators, also known as epigenetic clocks, use penalized regression models to predict chronological age based on DNA methylation levels (reviewed in [13, 14]). Methylation stands out from other genomic data in that it allows one to build pan-tissue clocks that apply to all tissue types across the entire lifespan (from prenatal samples to centenarians) $[11,12,15]$.

Species from the genus Peromyscus (deer mouse) are appealing models for addressing various biological questions in relation to aging because they live up to 8 years in captivity [16], a lifespan that exceeds by about threefold, that of animals of the genus Mus, the commonly used model for biomedical research. They are also used to study metabolism, infectious diseases, adaptation at extreme environments, such as high altitude and the desert, monogamous behavior, as well behavioral responses in response to anxiety stress [16-19]. In an effort to better understand how methylation profiles change in relation to different environments and genetic backgrounds, we have undertaken to study methylation patterns in the genus Peromyscus spanning different species and stocks/ populations. Peromyscus is comprised of species that were evolved in diverse range habitats, from deserts to high-altitude mountains, in environments with lowto-high extrinsic risk of predation. Thus, each one of these species gained several unique features to adapt, reproduce, and survive in these environments. Here, we present a comparative epigenetic analysis of these interesting characteristics. We characterize CpGs that relate to mating behavior (e.g., monogamy) and high/ low altitude.

Further, we present six highly accurate epigenetic clocks for Peromyscus. Two of these clocks apply to humans as well.

\section{Results}

Data sets

Different species of Peromyscus are maintained as closed colonies of outbred, genetically diverse stocks at the Peromyscus Genetic Stock Center. The present analysis involved 36,000 CpGs that are highly conserved across mammals in DNA and was applied to specimens from two Peromyscus subgenera, 5 species and one interspecific hybrid, 3 tissues (tails, brain, and liver), and individuals from ages ranging from 2 months to about 3.6 years. The choice of tissues was informed by several criteria. First, the Mammalian Methylation Consortium aims to collect liver samples and brain samples from as many species as possible for the sake of comparative studies. For these tissues, abundant methylation data are available from various species, allowing the cross-species evaluation of data generated. Second, we also aimed to profile a tissue that could be collected without sacrificing the animal (tail).

The specimens analyzed involved both males and females as well as individuals from two different closed colonies of $P$. maniculatus. This experimental setup facilitated a simultaneous, unbiased analysis of DNA methylation signatures in specimens differing across different levels of biological organization.

We used a custom methylation array (HorvathMammalMethylChip40) to generate DNA methylation data from six species of Peromyscus: Peromyscus californicus $(n=16)$, Peromyscus eremicus $(n=17)$, Peromyscus hybrid between $P$. polionotus and $P$. maniculatus $(n=6)$, Peromyscus leucopus $(n=36)$, Peromyscus maniculatus $(n=53)$, and Peromyscus polionotus $(n=16)$. Hierarchical clustering indicated the $n=6$ samples were technical outliers (Fig. 1). These samples were subsequently removed from the analysis. 


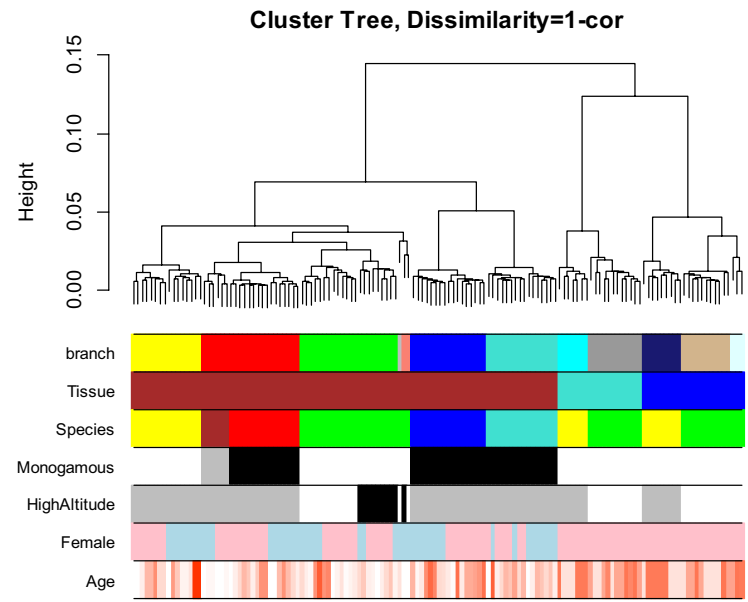

Fig. 1 Unsupervised hierarchical clustering of tissue samples from deer mice. Average linkage hierarchical clustering based on the interarray correlation coefficient (Pearson correlation). The first color band is based on cutting the branches at a height cutoff value of 0.03 . Note that the branch colors correspond to tissue (second color band) and species (third color band). Tissue encodes tail (brown), whole brain (turquoise), and liver (blue). Species encodes Peromyscus californicus (turquoise), eremicus (blue), leucopus (yellow), maniculatus (green), polionatus (red), and a hybrid between polionatus and maniculatus (brown). The fourth color band encodes monogamous (black) versus polygamous (white) behavior. The fifth color band encodes high altitude population (black) versus low altitude (white). Female (pink). Age color codes old (red) versus young (white)

These DNA samples came from three tissues/ organs: whole brain, tail, and liver as detailed in Table 1. The ages ranged from 0.083 to 3.6 years. Additionally, we used DNA methylation profiles from 1205 human samples, from several tissues, and with a large age range, to construct two dual-species
human-Peromyscus epigenetic clocks. These human data were generated on the same custom methylation array, which was designed to facilitate cross-species comparisons across mammals.

Unsupervised hierarchical clustering of deer mouse methylation data

Unsupervised hierarchical clustering of methylation data was initially performed that identified the tissue identity as the most prominent discriminator of global methylation signatures (Fig. 1). Thus, the profile of DNA methylation is primarily guided by a function which, in turn, underscores the impact of this epigenetic modification in regulating gene transcription and therefore guiding cellular differentiation. Within the same tissue, clustering occurred in a manner that overlapped astonishingly with the evolutionary history. Initially, two branches emerged, with the first including $P$. californicus and $P$. eremicus and the second comprised of $P$. leucopus, P. maniculatus, and $P$. polionotus, that signify two distinct groups in the evolution of Peromyscus [20]. Among them, the highly related $P$. maniculatus and $P$. polionotus clustered together, while $P$. leucopus has diverged earlier. Indeed, $P$. maniculatus and $P$. polionotus can form interspecific F1 hybrids that, in relation to their methylation patterns, are more closely related to the paternal strain, $P$. polionotus (Fig. 1) pointing to the impact of the parent of origin effects in guiding methylation signatures [21]. Among the same species, genetic relevance appeared to be of significance. Individuals from two closed $P$. maniculatus colonies were evaluated, bairdii and sonoriensis (BW and SM2 stocks, respectively) [16]. Individuals from
Table 1 Description of the data. $n=$ total number of tissues. Number of females. Age (in units of years): mean, minimum, and maximum age

\begin{tabular}{llllll}
\hline Species/tissue & $n$ & No. of females & Mean age & Min. age & Max. age \\
\hline Peromyscus californicus/tail & 16 & 7 & 0.955 & 0.083 & 2.75 \\
Peromyscus eremicus/tail & 17 & 9 & 1.36 & 0.16 & 2.66 \\
Hybrid polionotus + maniculatus/tail & 6 & 3 & 0.163 & 0.083 & 0.25 \\
Peromyscus leucopus/brain & 7 & 7 & 1.58 & 0.583 & 2.33 \\
Peromyscus leucopus/liver & 8 & 8 & 1.46 & 0.583 & 2.33 \\
Peromyscus leucopus/tail & 16 & 8 & 1.13 & 0.083 & 3.58 \\
Peromyscus maniculatus/brain & 12 & 12 & 1.58 & 0.583 & 2.42 \\
Peromyscus maniculatus/liver & 13 & 13 & 1.46 & 0.583 & 2.42 \\
Peromyscus maniculatus/tail & 25 & 14 & 0.724 & 0.083 & 2.83 \\
Peromyscus polionotus/tail & 16 & 9 & 0.909 & 0.083 & 1.91 \\
\hline
\end{tabular}


each of these colonies clustered accurately together suggesting that genetic relatedness is capable of triggering similar patterns of DNA methylation that surpass those inflicted by the sex and the age of the individuals from which the DNA samples have been isolated. Within the same species and stocks, clustering occurred according to sex. The fact that the analysis involved tails, whole brain, and liver samples that are not major target tissues for sex hormones implies that sex-specific patterns of methylation are inflicted early during development that persist at adulthood. Alternatively, the expression of receptors for gonadal steroids by these tissues may have caused modulation of the methylation profile and the observed clustering of the specimens according to sex; nevertheless, this appears to be of lesser impact as compared to the other variables examined.

\section{Epigenetic clocks}

Our different clocks can be distinguished along two dimensions (species and measure of age). The multitissue clock (also referred to as pan-tissue clock) for Peromyscus was developed by regressing chronological age on CpGs in all available tissue samples from all species. Since the regression model was fit to different Peromyscus species, the resulting age estimators are multi-species clocks that are expected to apply to all Peromyscus species. To arrive at unbiased estimates of our DNA methylation-based age estimators, we performed a cross-validation study in the training data. The cross-validation study reports unbiased estimates of the age correlation $R$ (defined as Pearson correlation between the age estimate (DNAm age) and chronological age as well as the median absolute error. The multi-tissue clock leads to a high correlation between estimated age and actual age (crossvalidation estimate of the Pearson correlation $R=0.9$, median absolute error $=0.24$ years $=3$ months, Fig. 2a). Similarly, we developed tissue-specific clocks by focusing on one specific tissue type. Since multiple Peromyscus species were used, the resulting clocks should again be interpreted as multispecies clocks. Cross-validation studies indicate high accuracy for the brain clock (cross-validation estimate $R=0.78$, MAE $=0.49$ years, Fig. 2b), liver clock $(R=0.94$, MAE $=0.20$, Fig. $2 \mathrm{c})$, and tail clock $(R=0.95$, MAE $=0.16$, Fig. $2 \mathrm{~d})$.
Evolutionarily speaking, primates are quite distant from Peromyscus. However, we managed to build two human-Peromyscus multi-tissue clocks. The first human-Peromyscus clock estimates chronological age using a single multivariate regression model. We observe high correlation coefficients between age and its estimates across both species (cross-validation estimate $R=0.99$, Fig. 2e) and when the analysis is restricted to Peromyscus samples $(R=0.79$, Fig. $2 \mathrm{f})$. The second human-Peromyscus clock estimates relative age, defined as the ratio of chronological age to maximum lifespan. This clock achieves a similar performance ( $R=0.97$ and $R=0.79$, Fig. $2 \mathrm{~g}, \mathrm{~h}$ ). By definition, the relative age takes values between 0 and 1 and arguably provides a biologically more meaningful comparison between species with different lifespans (deer mouse and human), which is not afforded by mere measurement of the absolute age.

Peromyscus are long-living rodents that exhibit variable lifespan depending on the species [22]. According to the database "anAge" [23], the maximum lifespans are as follows: 7.4 years for Peromyscus eremicus [24], 7.9 years for Peromyscus leucopus [24], 8.3 years for Peromyscus maniculatus [25], and 5.5 years for Peromyscus polionotus [24]. While the maximum lifespan estimates may be debatable, our clock is quite robust with respect to different choices of this mathematical parameter. Similarly, accurate clocks could be constructed with different parameter choices.

\section{EWAS of age}

In total, 29,125 probes from the mammalian chip (HorvathMammalMethylChip40) were aligned to specific loci approximate to 5048 genes in the deer mouse genome (Peromyscus_maniculatus_bairdii. HU_Pman_2.1.100). These probes have high conservation in mammals, thus comparable among different species. In our epigenome-wide association study (EWAS) of age, we correlated individual CpGs to chronological age. Stratified results for each deer mouse species and tissue type are presented in Supplementary Fig. 1. Significant age-related CpGs were located in both genic and intergenic regions relative to the transcriptional start site. CpGs located in promoters and $\mathrm{CpG}$ islands gained methylation with age. 

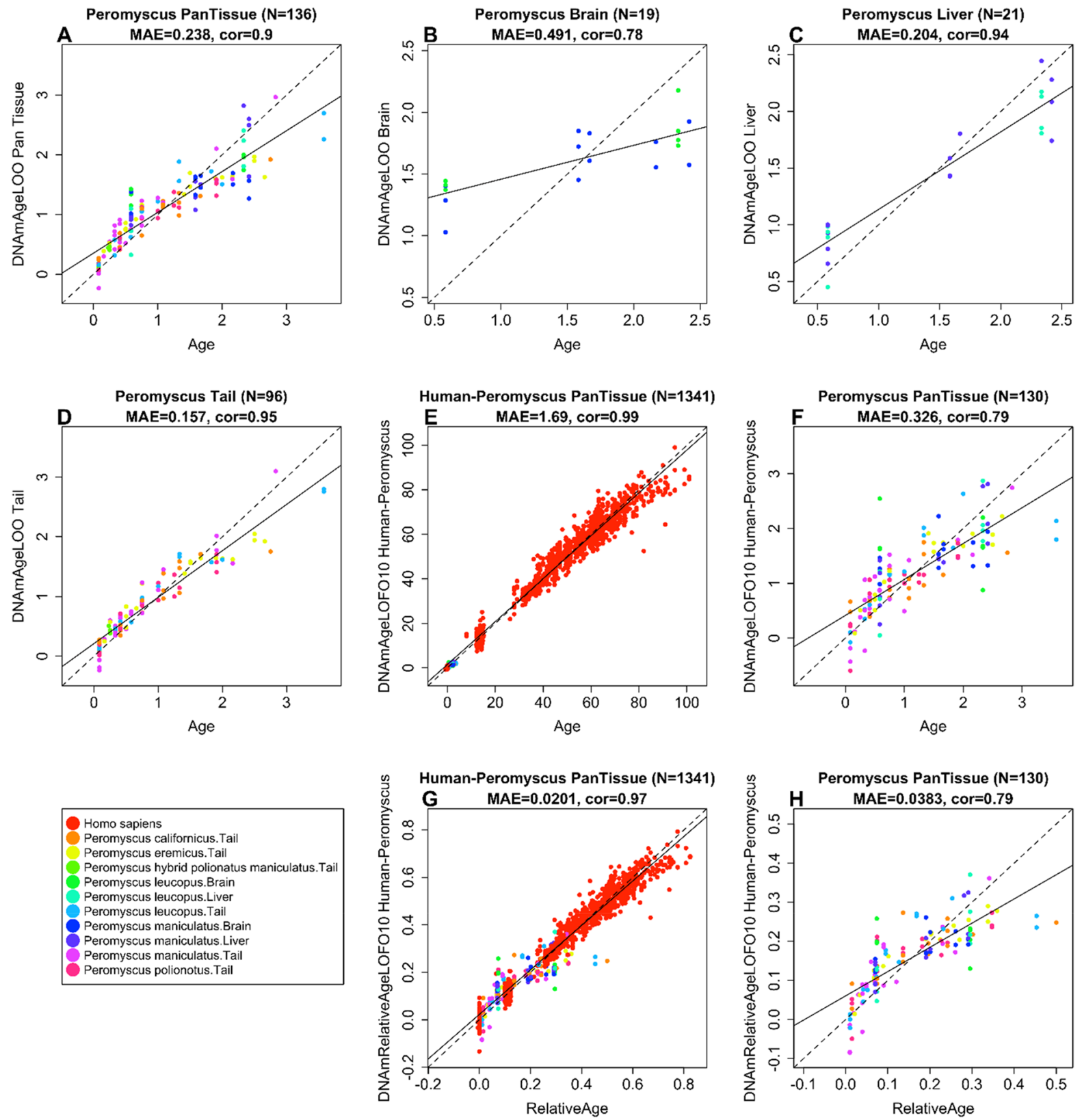

Fig. 2 Cross-validation study of epigenetic clocks for Peromyscus. The $y$-axis reports the DNA methylation estimate of $\mathbf{A}-\mathbf{F}$ chronological age (age in units of years) or $\mathbf{G}$ and $\mathbf{H}$ relative age. a Epigenetic clock for multiple Peromyscus species. Leave-one-sample-out (LOO) estimate of DNA methylation age (y-axis, in units of years) versus chronological age. A Multi-tissue clock for all considered Peromyscus species, B brain clock, $\mathbf{C}$ liver clock, and D tail clock. E Dual species
human-Peromyscus clock for chronological age, $\mathbf{F}$ excerpt of panel $\mathbf{E}$ restricted to Peromyscus samples. G Human-Peromyscus clock for relative age (defined as the ratio of chronological age to maximum lifespan). $\mathbf{H}$ Excerpt of panel $\mathbf{G}$ restricted to Peromyscus samples. Dots (DNA samples) are colored by species or tissue sample as outlined in the legend. Each panel reports the sample size, correlation coefficient, and median absolute error (MAE) 

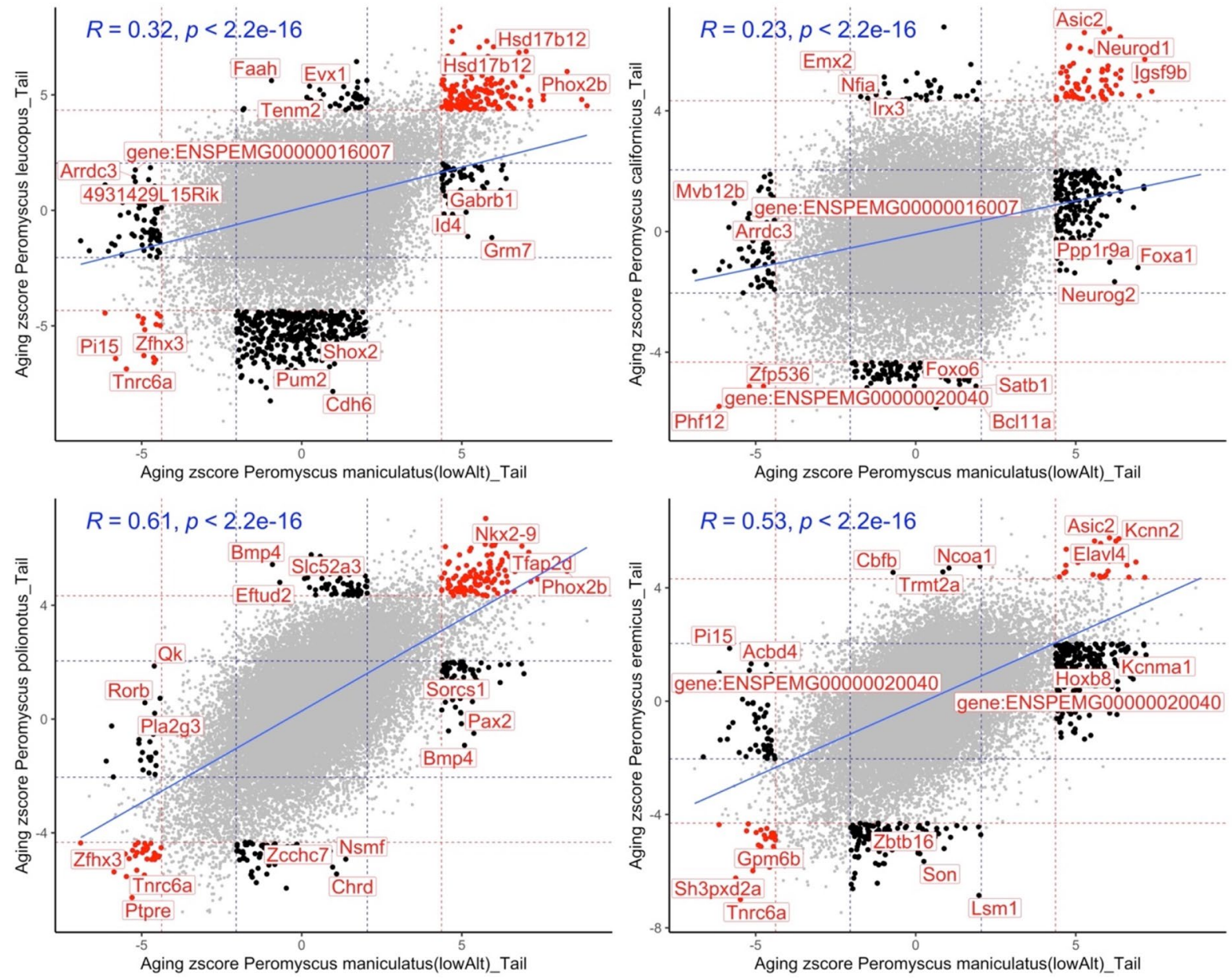

Fig. 3 Comparative DNAm aging between $P$. maniculatus and four other Peromyscus species. Scatter plots represent the EWAS of age in $P$. maniculatus ( $x$-axis) vs other four species. The aging $Z$ statistics are the Fisher $Z$-transformation of DNAm-age Pearson correlation for each $\mathrm{CpG}$ in the tail samples of each species. A positive (or decrease) of $Z$ statistics means an increase (or decrease) of DNAm by age in the analyzed species. Red dotted lines are the $Z$ statistics corresponding to $p<10^{-4}$; blue dotted lines are the $Z$ statistics corresponding to $p>0.05$; red dots indicate the shared CpGs

For comparative purposes, we contrasted these results to those from an EWAS of chronological age in liver and tail data from C57B1/6 mice (Mus musculus). We caution the reader that C57B1/6 mice are inbred, unlike our deer mice. In general, EWAS of age in tails and liver from Peromyscus species were only weakly related to the corresponding analyses of the same tissues from C57Bl/6 mice. Age effects

(i.e., The CpGs that significantly change in the same direction in both species) between $\mathrm{x}$ and $\mathrm{y}$ axes; black dots: the changes that are significant in one species but not the other. The top CpGs in each sector are labeled by the adjacent gene based on the P. maniculatus genome. Peromyscus maniculatus (lowAlt) is the P. maniculatus originated from the founders with a low altitude habitat. Sample size: Peromyscus maniculatus (lowAlt), 40; Peromyscus leucopus, 31; Peromyscus californicus, 16; Peromyscus polionotus, 16; Peromyscus eremicus, 17

in liver methylation data from C57B1/6 mice were only weakly correlated with those from $P$. maniculatus $(R=0.16$, Supplementary Fig. 3$)$ and $P$ leucopus $(R=0.32)$. Age effects in tail methylation data from $\mathrm{C} 57 \mathrm{Bl} / 6$ mice were only weakly correlated with those from $P$. maniculatus $(R=0.12$, Supplementary Fig. 4$),$ P leucopus $(R=0.47), P$. polionotus $(R=0.32)$. 
In a separate analysis, we compared epigenetic aging effects in $P$. maniculatus with those in four different Peromyscus species (Fig. 3). Pairwise correlations differed within these species which probably reflects evolutionary differences of these highly related species. Despite these differences, there were numerous CpGs with consistent aging patterns in the Peromyscus genome. Some of the shared changes in these animals included a gain of methylation in $B d n f$ (promoter), Igsf9b (exon), Nkx2-9 (downstream), Lhfpl4 (exon), Elavl4 (intron), and Trhde (promoter).

Epigenetic profile of North American deer mouse change along with the altitude

The present analysis involved individuals from two colonies of $P$. maniculatus sonoriensis (SM2 stock) and $P$. maniculatus bairdii (BW stock). The original founders of these colonies have evolved in high- or low-altitude environments, respectively, and since then are maintained as closed colonies. We identified a total of $1273 \mathrm{CpGs}$ that differed in their mean methylation levels at a nominal significance threshold of $p<10^{-4}$ (Fig. 4a).

CpGs in ENSPEMG00000020040 (downstream, $p=1 \times 10^{-26}$ ) and Madd (exon, $p=3 \times 10^{-17}$ ) show decreased methylation in the high-altitude stock P. maniculatus sonoriensis while $\mathrm{CpGs}$ in Nsd3 (intron), $P d z d 8$ (exon, $p=2.9 \times 10^{-22}$ ), and Stcl (promoter, $p=5 \times 10^{-20}$ ) show increased methylation (Fig. 4a). Functional enrichment studies of the top $500 \mathrm{CpGs}$ per direction (increased/decreased) showed that these loci tend to be located near genes that play a role in the development of rhombomere 3 $\left(p=3.9 \times 10^{-10}\right)$, motor neurons $\left(p=2 \times 10^{-7}\right)$, middle ear $\left(p=5 \times 10^{-10}\right)$, immune system functioning $\left(\mathrm{p}=2.8 \times 10^{-11}\right)$, antigen processing, and presentation $\left(\mathrm{p}=9.6 \times 10^{-6}\right)$.

Next, we examined the differences in aging patterns, i.e., differences in the age correlations between the two colonies of $P$. maniculatus. Aging effects showed a moderately high correlation between the two colonies ( $R=0.4$, Fig. $4 \mathrm{~b}, \mathrm{c})$, but several CpGs exhibited significant correlations only in one of the colonies. DNAm aging effect comparison by altitude was done at a nominal $p<10^{-3}$ in order to implicate sufficient numbers of $\mathrm{CpGs}$ for our subsequent enrichment studies.
For example, CpGs in the promoter of Nfia, upstream of Htatip2, in an exon of Mapre3, and an exon of $C x x 4$ correlated significantly with age only in the low altitude colony of $P$. maniculatus. Conversely, CpG upstream of Dlx6, an exon of Fam160a2, an intron of Fam241b, and upstream of Pax2 correlated significantly with age only in the high-altitude colony (Fig. 4d,e).

Interestingly, these altitude-specific age-related changes are adjacent to genes that play a role in brain development $\left(p=2.7 \times 10^{-4}\right)$, immune system functioning $\left(p=4.6 \times 10^{-5}\right)$, and T-cell development $\left(p=4.6 \times 10^{-5}\right)$. Overall, these results suggest that altitude affects both brain development and immune system functioning. Limitations of the analysis are discussed below.

Brain aging patterns in North American deer mouse species

Comparison of brain specimens between older P. leucopus and $P$. maniculatus indicated that in the latter, coordination of the unfolded protein response is compromised, and evidence of neurodegenerative pathology was obtained [26]. Therefore, comparative analysis of Peromyscus species may be relevant to the study of age-related alterations in the brain. Here, we compared the DNAm profile of the $P$. maniculatus brain to P. leucopus and found strong differences. A total number of $2396 \mathrm{CpGs}$ were differentially methylated between these two species at $p<10^{-4}$ (Fig. 5a). In $P$. maniculatus, CpGs were hypomethylated in Cadsp2 (exon, $p=1.3 \times 10^{-36}$ ), Caszl (exon, $p=3 \times 10^{-32}$ ) but hypermethylated in Grm8 (exon, $p=1.8 \times 10^{-35}$ ), Epha3 (promoter, $p=5 \times 10^{-33}$ ), and Lbxl (promoter, $\left.p=3.5 \times 10^{-31}\right)$. GREAT enrichment analysis of the top 500 most significant $\mathrm{CpGs}$ in each direction revealed that these $\mathrm{CpGs}$ were adjacent to genes that play a role in the circadian rhythm (GREAT hypergeometric $p=9 \times 10^{-12}$, Supplementary Fig. 6), histone H2A ubiquitination $\left(p=6 \times 10^{-8}\right)$, and glucagon secretion $\left(p=2.7 \times 10^{-7}\right)$. The latter finding surrounding glucagon secretion is interesting since several brain regions are known to be sensitive to glucagon by induction of cAMP signaling cascades [27], and insulin resistance may be related to Alzheimer's disease [28]. 


\section{Altitude effects on North American deer mouse (P.maniculatus) DNAm}

a

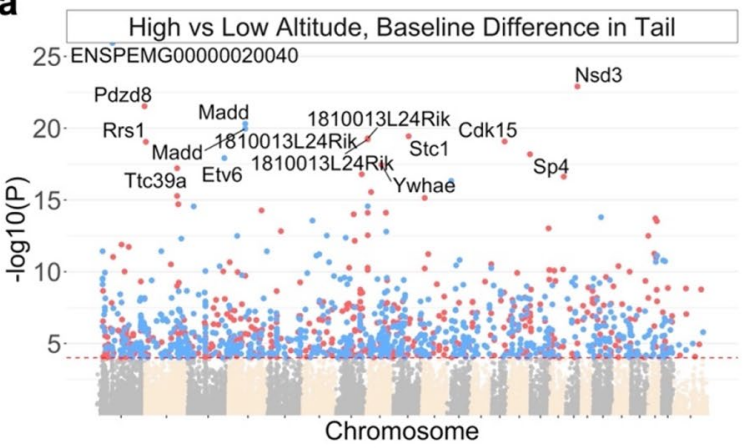

b Altitude effects on DNAm aging

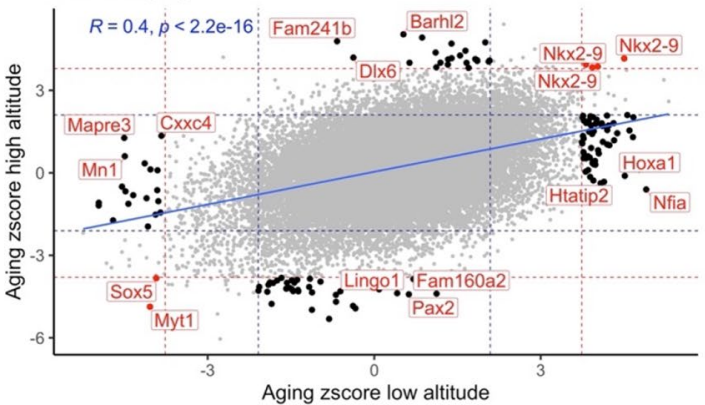

Altitude low high
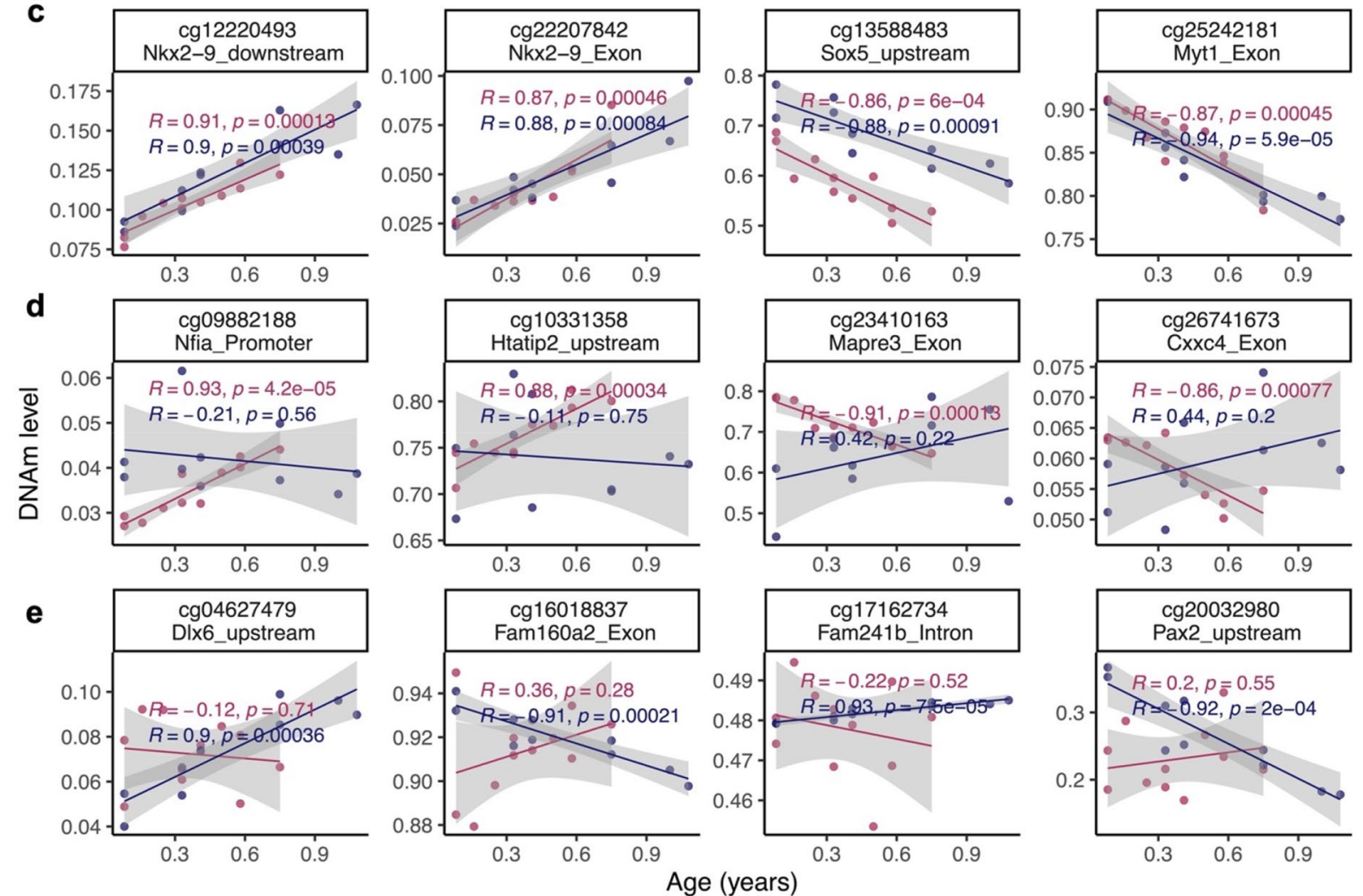

Fig. 4 Altitude difference altered epigenetic profiles of $P$. maniculatus. This study compares two colonies of $P$. maniculatus, which originated from the founders with different habitats in different altitudes. a The Manhattan plot of EWAS of altitude in the tail of $P$. maniculatus species after adjusting for chronological age. The genome coordinates are estimated based on the alignment of mammalian array probes to the Peromyscus_maniculatus_bairdii.HU_Pman_2.1.100 genome assembly. The direction of associations with $p<10^{-4}$ (red dotted line) is colored in red (increased methylation) and blue (decreased methylation). The 15 most significant CpGs are labeled by adjacent genes. b Sector plot of DNA methylation aging effects in $P$. maniculatus species from different alti- tudes. The $Z$ statistics for aging effects result from applying the Fisher $Z$-transformation to the Pearson correlation between age and the respective $\mathrm{CpG}$ in the tail samples of each colony. The red and blue dotted lines correspond to a $p$-value threshold of $p<0.01$ and $p<0.05$, respectively. Shared CpGs (i.e., the CpGs that significantly change in the same direction) are colorcoded in red. Black dots correspond to CpGs that are significant in one colony but not the other. The scatter plots represent the CpGs that change with age in both (c), or uniquely in low (d) or high (e) altitude colonies. The adjacent gene region of each $\mathrm{CpG}$ is reported in the title of each plot. The shade around the lines is the $95 \%$ confidence interval based on the linear regression model. $R$ : Pearson correlation coefficient 


\section{Brain differences between North American deer mouse (P.maniculatus) and white-footed mouse (P.leucopus)}
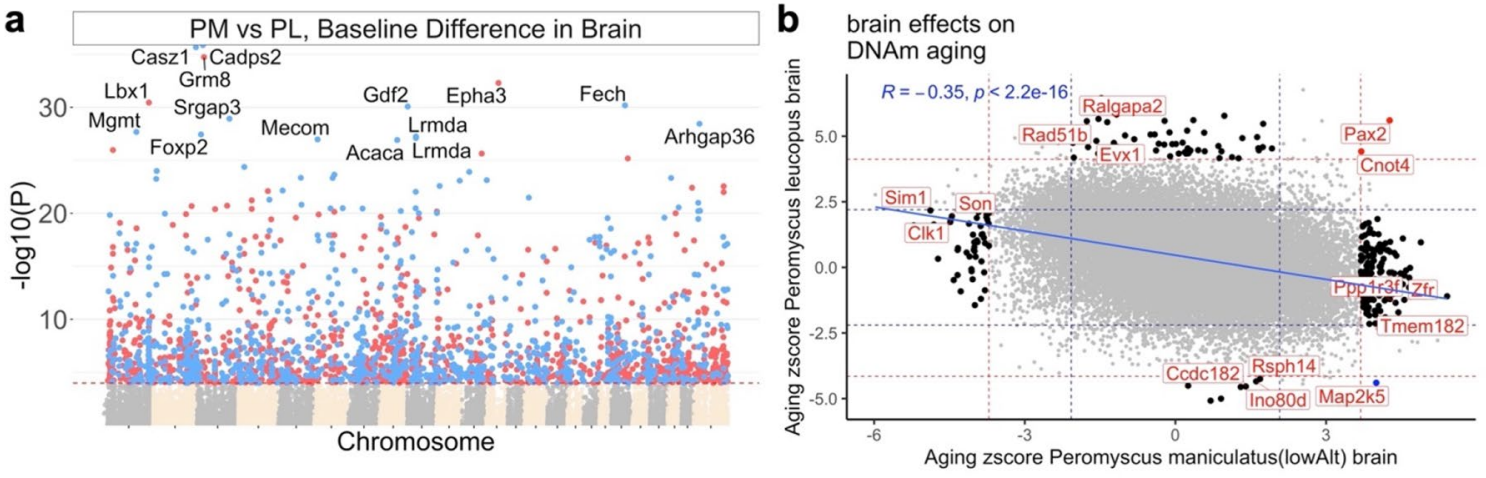

SpeciesLatinName Peromyscus maniculatus(lowAlt) Peromyscus leucopus

C
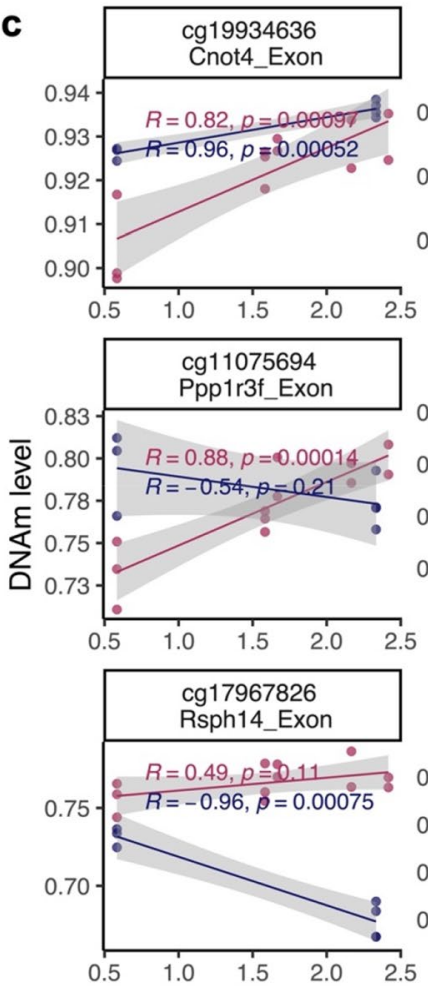
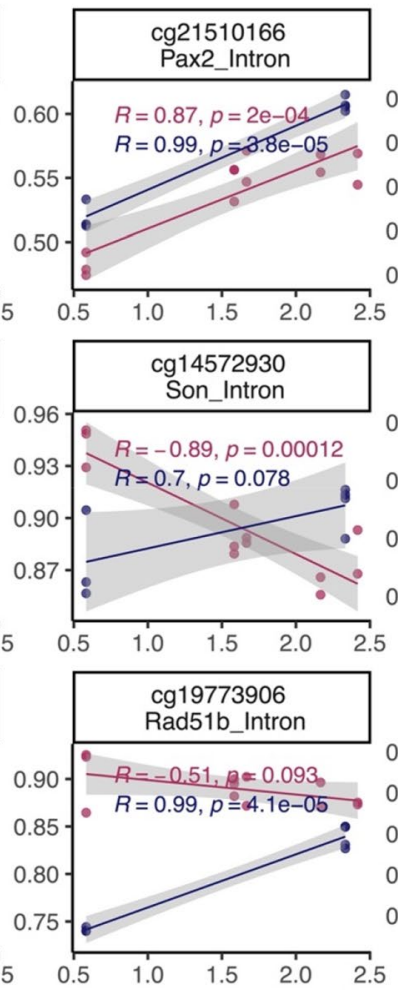
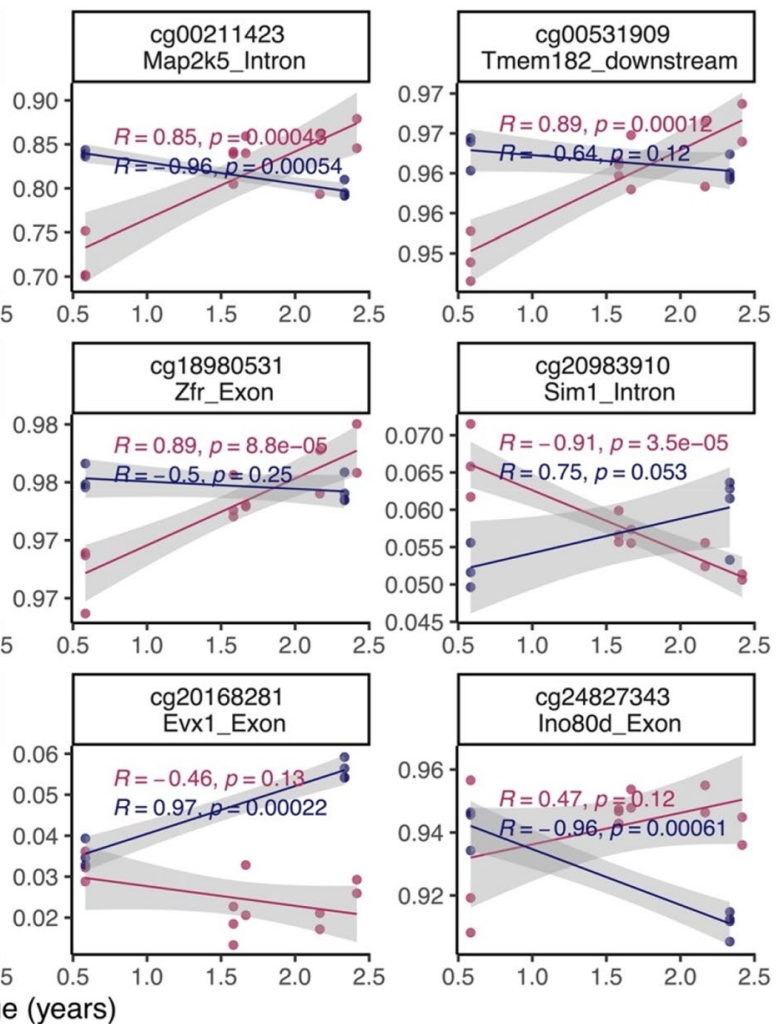

Fig. 5 Brain methylation differences between P. maniculatus (PM) and P. leucopus (PL). a The Manhattan plot of mean methylation differences between $P$. maniculatus and P. leucopus after adjusting for chronological age. Genome coordinates for the Peromyscus_maniculatus_bairdii.HU_Pman_2.1.100 genome assembly. The direction of associations with $p<10^{-4}$ (red dotted line) is colored in red (hypermethylated) or blue (hypomethylated). The top $15 \mathrm{CpGs}$ are labeled by adjacent genes. b Sector plot of DNAm aging effects in $P$. maniculatus and $P$. leucopus. The aging $Z$ statistics are the Fisher $Z$-transformation of DNAm-age Pearson correlation for each $\mathrm{CpG}$ in the tail samples of each species. The red and blue dotted lines correspond to a $p$-value threshold of $p<0.01$ and $p<0.05$, respectively. Red color codes shared $\mathrm{CpGs}$ (i.e., the $\mathrm{CpGs}$ that significantly change in the same direction) while black color code changes that are significant in one species but not the other. c The scatter plots represent the $\mathrm{CpG}$ that change with age in both or uniquely in one of these species. The adjacent gene region of each $\mathrm{CpG}$ is reported in the title of each plot. The shade around the lines is the $95 \%$ linear regression confidence interval. $R$ : Pearson correlation coefficient 
Strikingly, age effects in brain methylation data from $P$. maniculatus were inversely correlated $(R=-0.35)$ with those in $P$. leucopus brains (Fig. 5b). We could only identify two CpGs (adjacent to Cnot4 exon, Pax2 intron) that changed with age in the same direction in these species (Fig. 5c). In contrast, several brain aging loci were identified that only changed in one of these species, or even diverged during aging. Map $2 k 5$ intron was an extreme example that was hypermethylated in P. maniculatus but hypomethylated in P. leucopus (Fig. 5b,c). CpGs that were only significantly correlated with age in P. leucopus were enriched in angiogenesis-related processes $\left(p=2.3 \times 10^{-4}\right)$ while those unique to $P$. maniculatus were enriched in gamma delta T cells $\left(\mathrm{p}=9 \times 10^{-4}\right)$. However, the latter findings could be false positives since the enrichment $p$-values are not significant after adjusting for multiple comparisons (nominal $p$-value $>10^{-4}$ ).

DNAm relate to pair-bonding behavior in deer mouse species

Relatively, few mammalian species are monogamous [29]. Pair bonds based on mating, that are associated with the development of monogamous behavior are estimated to occur in less than $10 \%$ of mammals, including humans [30, 31]. In Peromyscus, monogamous behavior is fairly common and has developed independently at least twice in evolution [30, 32]. Our study involved three monogamous $(P$. californicus, $P$. polionotus, and $P$. eremicus) and two polygamous ( $P$. maniculatus and $P$. leucopus) species. Monogamous species differed greatly from polygamous species: $9411 \mathrm{CpGs}$ were differentially methylated at a nominal significance threshold of $p<10^{-4}$ (Fig. 6a). The most significant EWAS hits for monogamy included decreased methylation in Zeb2 intron $\left(p=1.8 \times 10^{-153}\right), 1700008$ P02Rik upstream $\left(p=6.2 \times 10^{-77}\right)$, Cadps intron $\left(p=4.1 \times 10^{-60}\right)$, and increased methylation in Fer exon $\left(p=9.2 \times 10^{-76}\right)$, Rnd3 exon $\left(p=1.24 \times 10^{-76}\right)$, and Srsf9 exon $\left(p=4.9 \times 10^{-41}\right)$ (Fig. 6b). For enrichment analysis, we limited the analysis to the top $500 \mathrm{CpGs}$ per direction (increased/decreased). While, a monogamous-related decrease of methylation was enriched with synaptic dopamine release $\left(p=8 \times 10^{-4}\right)$ in the mouse phenotype database, the increase of methylation was associated with immune-related biological processes such as antigen processing and presentation by MHCII cells $\left(p=1 \times 10^{-4}\right)$ (Supplementary Fig. 7). Dopamine seems to play a central role in pair bond formation, expression, and maintenance [33]. Zeb2 gene $\left(p=1.8 \times 10^{\times 153}\right)$, the top monogamyrelated gene in our analysis, is also a key regulator of midbrain dopaminergic neuron development [34].

\section{Discussion}

We expect that the availability of the human-Peromyscus epigenetic clocks will provide a significant boost to the attractiveness of Peromyscus as a biological model. This study provides a first step towards studying the epigenetic correlates of monogamous behavior and the effects of high altitude in Peromyscus.

The development of deer mouse epigenetic clocks described here was based on novel DNA methylation data that were derived from 3 deer mouse tissue types (brain, tail, and liver). The Peromyscus DNA methylation profiles reported here represent the most comprehensive dataset thus far of single-base resolution methylomes across multiple species, tissues, and ages.

The multi-tissue Peromyscus clock allows one to accurately estimate multiple tissue types from different Peromyscus species. This gives us confidence that these clocks will lead to high age correlations in new samples from other tissue types and from other Peromyscus species. However, differences in tissue type or species could lead to a constant offset (bias) between estimated age and actual age.

The two human-Peromyscus clocks estimate chronological and relative age, respectively. The dual-species clock for relative age demonstrates the feasibility of building epigenetic clocks for two species based on a single mathematical formula. These humanPeromyscus clocks also effectively demonstrate that epigenetic aging mechanisms are highly conserved between evolutionarily distant species. The fact that one can build such multispecies clocks reflects the technical properties of the mammalian methylation array platform (which focuses on conserved CpGs) [35] and biological properties of epigenetic aging effects that are highly conserved across mammals. Similar multispecies clocks have been constructed for many different mammalian species [36-41]. 
Fig. $6 \mathrm{CpGs}$ that differ between monogamous and polygamous Peromyscus species. a The Manhattan plot of the mean DNAm difference between monogamous and polygamous Peromyscus species after adjusting the analysis for chronological age in a tail sample of Peromyscus species. Monogamous species: $P$. californicus $(N=16), P$. polionotus $(N=16)$, P. eremicus $(N=16)$. Polygamous species: P. maniculatus (both altitude colonies, $N=25), P$. leucopus $(N=16)$. Genome coordinates from Peromyscus_maniculatus_bairdii. HU_Pman_2.1.100 genome assembly. The direction of associations with $p<10^{-3}$ (red dotted line) is colored in red (hypermethylated) and blue (hypomethylated). The top $15 \mathrm{CpGs}$ are labeled by their neighboring genes. b The box plot of the top $\mathrm{CpGs}$ that differed by dyadic relationship in Peromyscus species. The adjacent gene region of each $\mathrm{CpG}$ is reported in the title of each boxplot. The notches in the boxplots indicate the $95 \%$ confidence interval of the median DNAm values for each group a

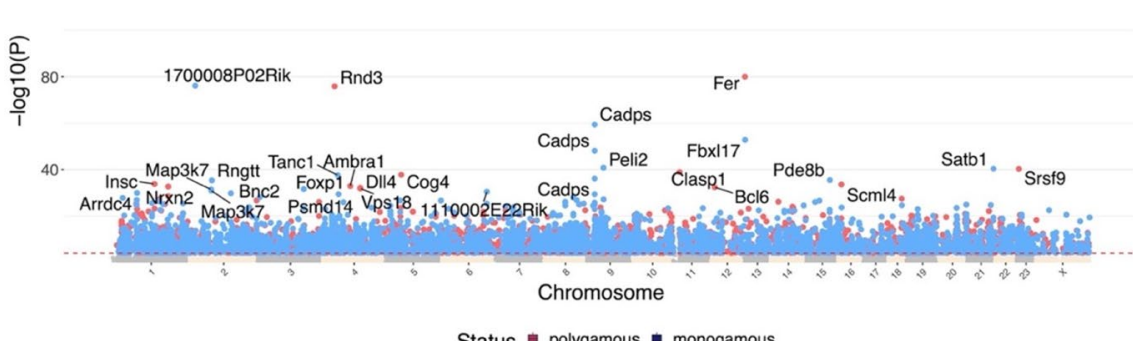

b

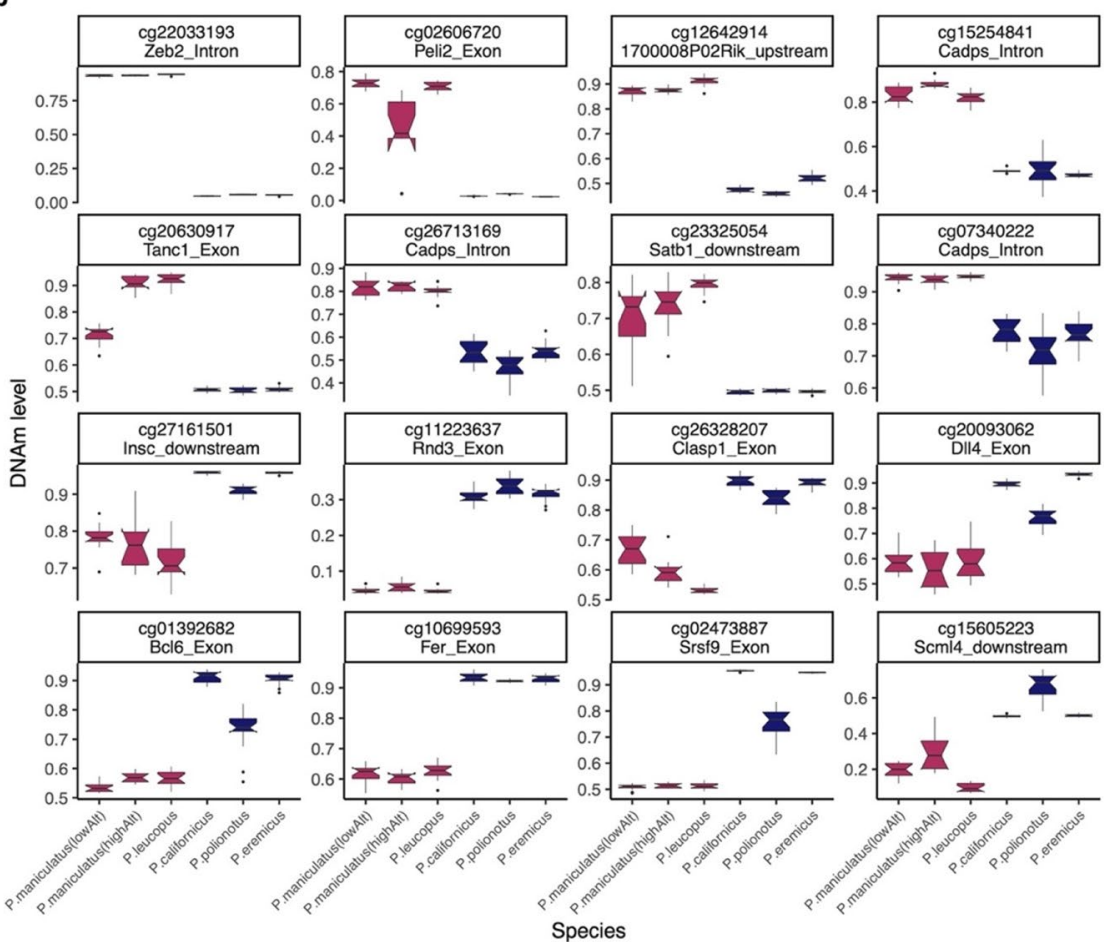

Our hierarchical clustering results reveal a robustly maintained hierarchical association of the biological variables (tissue, species) that can influence DNA methylation patterns. Tissue is a more important determinant of global methylation patterns than Peromyscus species (Fig. 1).

Our EWAS analyses identified CpGs associated with altitude at which the original founders of these colonies were captivated. These findings should be interpreted with caution because SM2 and BW stocks are highly diverse and are bred in isolation for extended periods which may have caused the fixation of methylation profiles that are irrelevant to the altitude adaptation of their original ancestors. Future studies should aim to replicate these results with wild-caught animals. While, however, no apparent differences have been recorded between the SM2 and the BW stocks that could associate with rhombomere 3 and motor neurons development, genes related to immune response and middle ear development may be of relevance. The latter may reflect adaptations associated with the differential atmospheric pressures at high altitudes. As regards the differential methylation of genes associated with immune system function, this may be relevant to the reported compromise of the immune system at high elevations [42-44]. To 
that end, high-altitude deer mice may have engaged epigenetic strategies to counteract such immune system suppression which has been linked, among others, to hypoxia at high elevations ${ }^{27}$. Of note is also the unique methylation profile of $P$. maniculatus in comparison with $P$. leucopus that corroborate the recently reported deregulation of stress response genes and the aberrant histological manifestations recorded in aged P. maniculatus [26].

Our EWAS of monogamy revealed strong methylation differences between monogamous and polygamous species. The fact that these results derived from tail tissues suggest that inherent differences in bonding behavior instruct specific epigenetic changes in peripheral tissues that may be translated into distinct physiological outcomes. Whether this is due to the differential regulation of specific neurohormonal circuits in response to hormones and neurotransmitters related to bonding, and which the exact physiological outputs are, remains to be determined.

Collectively, our study provided the first epigenetic clock for Peromyscus and illustrated the hierarchical association between various biological variables in determining methylation profiles across different scales of biological organization. Finally, it provided hints with regards to global differences and specific gene targets that are epigenetically impacted by biologically and environmentally relevant conditions.

\section{Materials and methods}

Deer mice are maintained as outbred, genetically diverse closed colonies in the Peromyscus Genetic Stock Center (PGSC) of the University of South Carolina. The specific stocks used were Peromyscus leucopus (LL stock, white-footed mice), Peromyscus eremicus (EP stock, cactus, or desert mice), Peromyscus polionotus (PO stock, oldfield mice) and Peromyscus manisculatus (North American deer mice). For P. maniculatus, in particular, animals from two stocks were used, $P$. maniculatus sonoriensis (SM2, high altitude) and P. manisculatus bardii (BW stock, low altitude). F1 hybrids between BW and PO stocks were also used. More details about the specific stocks are available in ref (9) and on the PGSC website (https:// go.sc.edu/pgsc). The study was approved by the Institutional Animal Care and Use Committee (IACUC) of the UofSC (protocol \#: 2356-101,506-042,720) and was in accordance with the guidelines set forth by the National Institutes of Health. DNA was isolated from live animals by tail snips, or upon sacrifice from livers and brains by using DNeasy DNA isolation kit (Qiagen) using the manufacturer's extraction. Our analysis is limited in that our brain data for P. leucopus and P. maniculatus derived from closed populations. The animals are technically outbred and genetic diversity is maintained, but there still may be a selection or drift over the several decades in captivity for/ toward phenotypes that would otherwise be disadvantageous in the wild. Strictly speaking, our conclusions are limited to these closed colonies, which have low effective population sizes.

Human tissue samples

To build the human-Peromyscus clock, we analyzed previously generated methylation data from $n=1205$ human tissue samples (adipose, blood, bone marrow, dermis, epidermis, heart, keratinocytes, fibroblasts, kidney, liver, lung, lymph node, muscle, pituitary, skin, spleen) from individuals whose ages ranged from 0 to 93 . These human tissue samples came from multiple sources: tissue and organ samples from the National NeuroAIDS Tissue Consortium [45], blood samples from the Cape Town Adolescent Antiretroviral Cohort study [46], and blood, skin, and other primary cells provided by Kenneth Raj [47] and blood samples from the PEG study [48]. Ethics approval was provided for all studies (IRB\#15-001,454, IRB\#16-000,471, IRB\#18-000,315, IRB\#16-002,028).

DNA methylation data

The DNA methylation data were generated using the mammalian methylation array (HorvathMammalMethylChip40) based on 37,492 CpG sites [35]. Not all of these CpGs apply to deer mice. In our analysis, we focused on 29,125 CpGs that are located near 5048 genes in the deer mouse genome (Peromyscus_ maniculatus_bairdii.HU_Pman_2.1.100).

Genome coordinates for each $\mathrm{CpG}$ are provided on the GitHub page of the Mammalian Methylation Consortium, see the section on data availability. The manifest file of the mammalian methylation array can be found at Gene Expression Omnibus (GEO) at NCBI as platform GPL28271. The SeSAMe normalization 
method was used to define beta values for each probe [49].

We caution the reader that small differences in sequences across species could have an effect on methylation levels.

To find technical outliers, we applied unsupervised hierarchical clustering (average linkage), which used 1 minus the interarray Pearson correlation as a dissimilarity measure. This analysis implicated five putative outliers which were severely outlying (i.e., that did not correlate with other samples from the same group). To err on the side of caution, we removed these samples from our analysis.

\section{Penalized regression models}

Details on the clocks (CpGs, genome coordinates) and R software code are provided in the Supplement. Penalized regression models were created with glmnet [50]. We investigated models produced by both "elastic net" regression (alpha $=0.5$ ). The optimal penalty parameters in all cases were determined automatically by using tenfold internal cross-validation (cv.glmnet) on the training set. By definition, the alpha value for the elastic net regression was set to 0.5 (midpoint between Ridge and Lasso type regression) and was not optimized for model performance.

We performed a cross-validation scheme for arriving at unbiased (or at least less biased) estimates of the accuracy of the different DNAm-based age estimators. One type consisted of leaving out a single sample (LOOCV) from the regression, predicting an age for that sample, and iterating over all samples. A critical step is the transformation of chronological age (the dependent variable).

Details on the clocks (CpGs, genome coordinates), coefficient values, and age transformations are provided in the Supplement.

To introduce biological meaning into age estimates of deer mice and humans that have a very different lifespan, as well as to overcome the inevitable skewing due to unequal distribution of data points from deer mice and humans across the age range, relative age estimation was made using the formula: relative age $=$ age/maxlifespan where the maximum lifespan for the two species was chosen from the an age data base [23]. We used the following maximum lifespans Peromyscus californicus (5.5 years), Peromyscus eremicus (7.4 years), Peromyscus leucopus (7.9 years),
Peromyscus maniculatus (8.3 years), Peromyscus polionotus (5.5 years), and humans (122.5 years), respectively.

Epigenome-wide association studies of age

EWAS was performed in each tissue separately using the $\mathrm{R}$ function "standardScreeningNumericTrait" from the "WGCNA" R package [51]. We used Stouffer's meta-analysis method to combine aging effects across different tissue types. Stouffer's method forms a linear combination of the $Z$-scores which are calculated in each stratum (e.g., based on tissue type) [52]. We chose the same weight for each tissue type.

\section{GREAT analysis}

We analyzed gene set enrichments using GREAT [53]. The GREAT enrichment analysis automatically conditioned out (removed) any bias resulting from the design of the mammalian array by using a background set of $\mathrm{CpGs}$ that map to horses and are located on the mammalian array. The GREAT software performs both a binomial test (over genomic regions) and a hypergeometric test over genes.

We performed the enrichment based on default settings (proximal: $50.0 \mathrm{~kb}$ upstream, $1.0 \mathrm{~kb}$ downstream, plus distal: up to $1000 \mathrm{~kb}$ ) for gene sets implemented in GREAT. To avoid large numbers of multiple comparisons, we restricted the analysis to the gene sets with between 10 and 3000 genes. We report nominal $p$-values and two adjustments for multiple comparisons: Bonferroni correction and the Benjamini-Hochberg false discovery rate.

Acknowledgements This work was supported by the Paul G. Allen Frontiers Group (PI Horvath) and by the NSF (OIA1736150) (PI H. Kiaris). The PGSC is supported by a grant from the NSF (DBI-1755670) (PI H. Kiaris). The funders had no role in the study design, data collection and analysis, decision to publish, or preparation of the manuscript.

Author contribution Steve Horvath and Amin Haghani are joint first authors of the study.

Data availability The data will be made publicly available as part of the data release from the Mammalian Methylation Consortium. Genome annotations of these CpGs can be found on Github https://github.com/shorvath/MammalianMethylation Consortium. 


\section{Declarations}

Conflict of interest SH is a founder of the nonprofit Epigenetic Clock Development Foundation which plans to license several patents from his employer UC Regents. These patents list $\mathrm{SH}$ as the inventor. The other authors declare no conflicts of interest.

Open Access This article is licensed under a Creative Commons Attribution 4.0 International License, which permits use, sharing, adaptation, distribution and reproduction in any medium or format, as long as you give appropriate credit to the original author(s) and the source, provide a link to the Creative Commons licence, and indicate if changes were made. The images or other third party material in this article are included in the article's Creative Commons licence, unless indicated otherwise in a credit line to the material. If material is not included in the article's Creative Commons licence and your intended use is not permitted by statutory regulation or exceeds the permitted use, you will need to obtain permission directly from the copyright holder. To view a copy of this licence, visit http://creativecommons.org/licenses/by/4.0/.

\section{References}

1. Smith ZD, Meissner A. DNA methylation: roles in mammalian development. Nat Rev Genet. 2013;14:204-20.

2. Cedar H. DNA methylation and gene activity. Cell. 1988;53:3-4.

3. Hannon E, Knox O, Sugden K, Burrage J, Wong CCY, Belsky DW, et al. Characterizing genetic and environmental influences on variable DNA methylation using. PLOS Gen. 2018;14:e1007544.

4. Teschendorff AE, Menon U, Gentry-Maharaj A, Ramus SJ, Weisenberger DJ, Shen H, et al. Age-dependent DNA methylation of genes that are suppressed in stem cells is a hallmark of cancer. Genome Res. 2010;20:440-6.

5. Jung M, Pfeifer GP. Aging and DNA methylation. BMC Biol. 2015;13:1-8.

6. Rakyan VK, Down TA, Maslau S, Andrew T, Yang TP, Beyan $\mathrm{H}$, et al. Human aging-associated DNA hypermethylation occurs preferentially at bivalent chromatin domains. Genome Res. 2010;20:434-9.

7. Horvath S. DNA methylation age of human tissues and cell types. Genome Biol. 2013;14:R115.

8. Petkovich DA, Podolskiy DI, Lobanov AV, Lee SG, Miller RA, Gladyshev VN. Using DNA methylation profiling to evaluate biological age and longevity interventions. Cell Metab. 2017;25:954-60 (e6).

9. Wang T, Tsui B, Kreisberg JF, Robertson NA, Gross AM, $\mathrm{Yu}$ MK, et al. Epigenetic aging signatures in mice livers are slowed by dwarfism, calorie restriction and rapamycin treatment. Genome Biol. 2017;18:57.

10. Stubbs TM, Bonder MJ, Stark AK, Krueger F, von Meyenn F, Stegle O, et al. Multi-tissue DNA methylation age predictor in mouse. Genome Biol. 2017;18:68.

11. Thompson MJ, Chwialkowska K, Rubbi L, Lusis AJ, Davis RC, Srivastava A, et al. A multi-tissue full lifespan epigenetic clock for mice. Aging (Albany NY). 2018;10:2832-54.

12. Meer MV, Podolskiy DI, Tyshkovskiy A, Gladyshev VN. A whole lifespan mouse multi-tissue DNA methylation clock. eLife. 2018;7:e40675.

13. Horvath S and Raj K, "DNA methylation-based biomarkers and the epigenetic clock theory of ageing," Nat Rev Genet, 2018.

14. Bell CG, Lowe R, Adams PD, Baccarelli AA, Beck S, Bell JT, et al. DNA methylation aging clocks: challenges and recommendations. Genome Biol. 2019;20:249.

15. Horvath S. DNA methylation age of human tissues and cell types. Genome Biol. 2013; 14(10):R115. https://doi. org/10.1186/gb-2013-14-10-r115

16. Havighorst A, Crossland J, Kiaris H. Peromyscus as a model of human disease. Semin Cell Dev Biol. 2017;61:150-5.

17. Steinman MQ, Trainor BC. Sex differences in the effects of social defeat on brain and behavior in the California mouse: insights from a monogamous rodent. Semin Cell Dev Biol. 2017;61:92-8.

18. Bendesky A, Kwon YM, Lassance JM, Lewarch CL, Yao S, Peterson BK, et al. The genetic basis of parental care evolution in monogamous mice. Nature. 2017;544:434-9.

19. Storz JF, Sabatino SJ, Hoffmann FG, Gering EJ, Moriyama H, Ferrand N, et al. The molecular basis of high-altitude adaptation in deer mice. PLoS Genet. 2007;3:e45.

20. Bedford NL, Hoekstra HE. Peromyscus mice as a model for studying natural variation. Elife. 2015;4:e06813.

21. Vrana PB, Guan XJ, Ingram RS, Tilghman SM. Genomic imprinting is disrupted in interspecific Peromyscus hybrids. Nat Genet. 1998;20:362-5.

22. Vrana PB, Shorter KR, Szalai G, Felder MR, Crossland JP, Veres M, et al. Peromyscus (deer mice) as developmental models. Wiley Interdiscip Rev Dev Biol. 2014;3:211-30.

23. de Magalhaes JP, Costa J, Church GM. An analysis of the relationship between metabolism, developmental schedules, and longevity using phylogenetic independent contrasts. J Gerontol A Biol Sci Med Sci. 2007;62:149-60.

24. Weigl R, "Longevity of mammals in captivity; from the living collections of the world," 2005.

25. Nowak RM, Walker EPR. Walker's mammals of the world. 6th ed. Baltimor: Johns Hopkins University Press; 1999.

26. Soltanmohammadi E, Farmaki E, Zhang Y, Naderi A, Kaza V, Chatzistamou I, et al. Coordination in the unfolded protein response during aging in outbred deer mice. Exp Gerontol. 2021;144:111191.

27. Abraham MA, Lam TKT. Glucagon action in the brain. Diabetologia. 2016;59:1367-71.

28. Kellar D, Craft S. Brain insulin resistance in Alzheimer's disease and related disorders: mechanisms and therapeutic approaches. Lancet Neurol. 2020;19:758-66.

29. Klug H. Why monogamy? A review of potential ultimate drivers. Front Ecol Evol. 2018;6:30.

30. Kleiman DG. Monogamy in mammals. Q Rev Biol. 1977;52:39-69.

31. Scribner JL, Vance EA, Protter DSW, Sheeran WM, Saslow E, Cameron RT, et al. A neuronal signature 
for monogamous reunion. Proc Natl Acad Sci USA. 2020;117:11076-84.

32. Jašarević E, Bailey DH, Crossland JP, Dawson WD, Szalai G, Ellersieck MR, et al. Evolution of monogamy, paternal investment, and female life history in Peromyscus. J Comp Psychol. 2013;127:91-102.

33. Curtis JT, Liu Y, Aragona BJ, Wang Z. Dopamine and monogamy. Brain Res. 2006;1126:76-90.

34. Hegarty SV, Wyatt SL, Howard L, Stappers E, Huylebroeck D, Sullivan AM, et al. Zeb2 is a negative regulator of midbrain dopaminergic axon growth and target innervation. Sci Rep. 2017;7:8568.

35. Arneson A, Haghani A, Thompson MJ, Pellegrini M, Kwon SB, $\mathrm{Vu} \mathrm{H}$, et al., "A mammalian methylation array for profiling methylation levels at conserved sequences," bioRxiv, p. 2021.01.07.425637, 2021.

36. Schachtschneider KM, Schook LB, Meudt JJ, Shanmuganayagam D, Zoller JA, Haghani A, et al., Epigenetic clock and DNA methylation analysis of porcine models of aging and obesity," GeroScience, 2021.

37. Horvath S, Zoller JA, Haghani A, Jasinska AJ, Raj K, Breeze CE, et al., "Epigenetic clock and methylation studies in the rhesus macaque," GeroScience, 2021.

38. Horvath S, Zoller JA, Haghani A, Lu AT, Raj K, Jasinska $\mathrm{AJ}$, et al., "DNA methylation age analysis of rapamycin in common marmosets," GeroScience, 2021.

39. Raj K, Szladovits B, Haghani A, Zoller JA, Li CZ, Black $\mathrm{P}$, et al., "Epigenetic clock and methylation studies in cats," GeroScience, 2021.

40. Sugrue VJ, Zoller JA, Narayan P, Lu AT, Ortega-Recalde OJ, Grant MJ, et al. Castration delays epigenetic aging and feminizes DNA methylation at androgen-regulated loci. eLife. 2021;10:e64932.

41. Prado NA, Brown JL, Zoller JA, Haghani A, Yao M, Bagryanova LR, et al. Epigenetic clock and methylation studies in elephants. Aging Cell. 2021;20:e13414.

42. Mazzeo RS. Altitude, exercise and immune function. Exerc Immunol Rev. 2005;11:6-16.

43. Oliver SJ, Macdonald JH, Harper Smith AD, Lawley JS, Gallagher CA, Di Felice U, et al. High altitude impairs in vivo immunity in humans High Altitude. Med Biol. 2013;14:144-9.

44. Meehan RT. Immune suppression at high altitude. Ann Emerg Med. 1987;16:974-9.
45. Morgello S, Gelman B, Kozlowski P, Vinters H, Masliah E, Cornford M, et al. The National NeuroAIDS Tissue Consortium: a new paradigm in brain banking with an emphasis on infectious disease. Neuropathol Appl Neurobiol. 2001;27:326-35.

46. Horvath S, Stein DJ, Phillips N, Heany SJ, Kobor MS, Lin DTS, et al. Perinatally acquired HIV infection accelerates epigenetic aging in South African adolescents. AIDS (London, England). 2018;32:1465-74.

47. Kabacik S, Horvath S, Cohen H, Raj K. Epigenetic ageing is distinct from senescence-mediated ageing and is not prevented by telomerase expression. Aging (Albany NY). 2018;10:2800-15.

48. Horvath S, Ritz BR. Increased epigenetic age and granulocyte counts in the blood of Parkinson's disease patients. Aging (Albany NY). 2015;7:1130-42.

49. Zhou W, Triche TJ Jr, Laird PW, Shen H. SeSAMe: reducing artifactual detection of DNA methylation by Infinium BeadChips in genomic deletions. Nucleic Acids Res. 2018;46:e123-e123.

50. Friedman J, Hastie T, Tibshirani R. Regularization Paths for generalized linear models via coordinate descent. J Stat Softw. 2010;33:1-22.

51. Langfelder P, Horvath S. WGCNA: an R package for weighted correlation network analysis. BMC Bioinformatics. 2008;9:559.

52. Stouffer SA, Suchman EA, DeVinney LC, Star SA, and Williams RM Jr, The American soldier: adjustment during army life.(studies in social psychology in World War II). 1 1949.

53. McLean CY, Bristor D, Hiller M, Clarke SL, Schaar BT, Lowe CB, et al. GREAT improves functional interpretation of cis-regulatory regions. Nat Biotechnol. 2010;28:495-501.

Publisher's note Springer Nature remains neutral with regard to jurisdictional claims in published maps and institutional affiliations. 